\title{
ESQUISTOSSOMOSE MANSÔNICA: UMA ANÁLISE DO PERFIL EPIDEMIOLÓGICO NA REGIÃO SUDESTE
}

\author{
SCHISTOSOMIASIS MANSONI: AN ANALYSIS IN OF THE EPIDEMIOLOGICAL \\ PROFILE IN THE SOUTHEAST REGION
}

João Victor Barreto Costa, José Marques da Silva Filho

Universidade Federal Fluminense

Abstract

This study aims to trace the epidemiological profile of schistosomiasis mansoni (SM) in Southeast region of Brazil between the years 2013 to 2017. It is a type of descriptive study of temporal trend, in which data available in the notification records of the Informatics Department of the Unified Health System (DATASUS) were used, considering the period from 2013 to 2017. The calculation of incidence was obtained through the population estimate projected by the Brazilian Institute of Geography and Statistics - IBGE. The data collected were processed by Excel 2016. During the period 21,464 cases of the disease were reported in Southeastern Brazil, with Minas Gerais (73.25\%) and São Paulo (15.52\%) having the highest absolute values of contamination. It is noticed that the incidence in the region decreased from 6.03 to 3.35 cases / year. The highest frequency of cases involved adults between the ages of 20 and 39, with the male sex (35.5\%) being the most affected. Moreover, MS was more prevalent in the urban area (72.7\%). Thus, the reported cases and the incidence of the disease decreased in the studied region. It is concluded that preventive and educational strategies need to be adopted, in order to reduce the incidence of MS in the endemic area of the Southeast region.

Keywords: Schistosoma mansoni. Schistosomiasis. Neglected Diseases. Brazil.
Resumo

Este estudo tem o objetivo de traçar o perfil epidemiológico da esquistossomose mansônica (EM) na região Sudeste do Brasil entre os anos de 2013 a 2017. É um tipo de estudo descritivo de tendência temporal, em que foram utilizados dados disponíveis nos registros de notificação do Departamento de Informática do Sistema Único de Saúde (DATASUS), considerando o período de 2013 a 2017. O cálculo da incidência foi obtido através da estimativa populacional projetada pelo Instituto Brasileiro de Geografia e Estatística - IBGE. Os dados coletados foram processados pelo Excel 2016. Durante o período foram notificados 21.464 casos da doença no Sudeste brasileiro, tendo Minas Gerais (73,25\%) e São Paulo (15,52\%) os maiores valores absolutos de contaminação. Percebe-se que a incidência na região diminuiu de 6,03 para 3,35 casos/ano. A maior frequência dos casos envolveu os adultos que estão entre a faixa etária dos 20 aos 39 anos, sendo o sexo masculino (35,5\%) o mais acometido. Além disso, a EM teve uma maior predominância na zona urbana (72,7\%). Assim, os casos notificados e a incidência da doença diminuíram na região estudada. Conclui-se que estratégias preventivas e educacionais necessitam ser adotadas, com o intuito de reduzir a incidência de EM na área endêmica da região Sudeste.

Palavras-chave: Schistosoma mansoni. Esquistossomose. Doenças negligenciadas. Brasil. 
A esquistossomose é uma doença infecto-parasitária desencadeada por trematódeos pertencentes ao gênero Schistosoma, sendo S. mansoni a única espécie encontrada nas Américas que acarreta danos ao ser humano pela ausência de hospedeiros intermediários específicos para as outras espécies de Schistosoma ${ }^{1,2}$. No Brasil, essa enfermidade recebe diversas sinonímias como "barriga d'agua", "mal do caramujo" ou "xistose" ${ }^{3}$, ocasionando agravos tanto na saúde pública nacional quanto internacional ${ }^{4}$.

Numa escala global, em 2018, foi estimado que 229,4 milhões de pessoas encontram-se com esquistossomose, das quais apenas 97,5 milhões receberam o tratamento preventivo ${ }^{5}$. Essa doença tropical negligenciada (DNT) de distribuição mundial é relatada em 78 países $^{5,6}$ e, atualmente, mais de 700 milhões de indivíduos habitam nas áreas endêmicas ${ }^{7}$. Em paralelo, o continente americano contém 25 milhões de pessoas que estão sob o risco de infecção ${ }^{8}$ e estima-se que $S$. mansoni infecta cerca de 1,5 milhões de habitantes brasileiros ${ }^{9}$.

Portanto, a transmissão dessa parasitose ocorre por mecanismo ativocutâneo, pelo contato do indivíduo com água doce contaminada com cercárias ${ }^{10}$. É importante ressaltar que a luminosidade, a temperatura e o oxigênio necessitam estar em níveis adequados para o desenvolvimento do parasito. Inúmeros fatores estão associados ao avanço dessa enfermidade, como questões socioeconômicas, ambientais, políticas e comportamentais, além das atividades relacionadas ao ecoturismo $3,11,12,13,14$. A falta de saneamento básico e do tratamento de esgoto ocasionam o deságue do conteúdo fecal contaminado para algumas coleções hídricas ${ }^{1}$. Além disso, os períodos chuvosos causam o aumento dos criadouros de moluscos Biomphalaria ssp. que consequentemente, favorecem a propagação do parasito ${ }^{15}$. Dessa forma, muitos homens, mulheres e crianças estão propícios a infecção, pois muitas atividades diárias levam o indivíduo a exposição a águas contaminadas ${ }^{3}$.

O ciclo biológico do $S$. mansoni depende da passagem desse helminto por dois hospedeiros, o intermediário e o definitivo. 0 hospedeiro intermediário é representado pelo caramujo do gênero Biomphalaria que é responsável pela reprodução assexuada do parasito. No território brasileiro, comprova-se a existência de três espécies desse molusco que são infectadas naturalmente pelo $S$. mansoni, sendo elas: $B$. glabrata, $B$. tenagophila e $B$. straminea. Já o ser humano é o principal hospedeiro definitivo desse helminto, no qual se desenvolve na sua forma madura, permitindo a oviposição das fêmeas ${ }^{1,2}$.

Em seu ciclo evolutivo, após a maturação sexual e o acasalamento de $S$. mansoni no sistema porta, ocorre a migração dos helmintos adultos para o sistema venoso do intestino, principalmente, para os vasos mesentéricos inferior e seus ramos ${ }^{16}$. Nesses vasos, há a deposição e a extrusão dos ovos em direção a luz intestinal, sendo posteriormente eliminados nas fezes. As condições precárias do local e a falta de educação sanitária predispõem para que as fezes contaminadas sejam despejadas em coleções aquáticas. A eclosão sucede do contato desses ovos com a água, liberando o miracídio do seu interior para o meio externo que, por meio de mecanismos intrínsecos, penetra nos tecidos dos caramujos transformando-se em esporocistos. Semanas seguintes, os esporocistos produzem e liberam a forma larvar do parasito, conhecidas como cercárias, que infectam o ser humano através da pele ou mucosas. Após penetração, a cercaria origina o esquistossômulo que, por meio da circulação sistêmica, chega ao sistema porta-hepático, evoluindo em helminto adulto e, portanto, reiniciando o seu ciclo ${ }^{3,17}$.

A esquistossomose mansônica (EM) caracteriza-se por manifestações clínicas variáveis de sintomatologia aguda ou crônica, que depende da intensidade da carga parasitária que o indivíduo apresenta. A forma aguda pode vir acompanhada de casos assintomáticos, presente em parte dos infectados, ou de quadros de dermatite cercariana e sinais e sintomas comuns de outras helmintoses intestinais, como diarreia muco-sanguinolenta, dores abdominais, febre e vômitos. A hepatoesplenomegalia, a hipertensão pulmonar, a ascite e as varizes esofagianas originada a partir do aumento da pressão portal são alguns dos sinais que são encontrados em pacientes na fase crônica ${ }^{1,2,3}$.

No diagnóstico clínico é importante abordar a história social do paciente e atentarse para sinais e sintomas típicos de esquistossomose, já que as manifestações clínicas podem se assemelhar com a de outras doenças ${ }^{18}$. Diante disso, o diagnóstico laboratorial é essencial, sendo o exame coproparasitológico um dos principais testes 
avaliativos, buscando-se a presença de ovos nas fezes. A técnica de Hoffman tem como finalidade a sedimentação espontânea do material fecal, apresentando-se como um método qualitativo e de alta sensibilidade ${ }^{2}$. Já o método de Kato-Katz, segundo a Organização Mundial da Saúde (OMS) é o mais recomendado para o diagnóstico da doença e tem como princípio a quantificação de ovos por grama de fezes ${ }^{6,19,20}$, porém estudos recentes apontam a limitação da técnica para detectar infecções de baixa intensidade ${ }^{21,22,23}$. Em relação ao tratamento específico para a redução da carga parasitária e a possível cura, são utilizados os fármacos praziquantel e o oxamniquina, ambos disponíveis no Sistema Único de Saúde ${ }^{24}$.

A região Sudeste é a mais populosa e povoada do Brasil, e abriga no seu território várias espécies do hospedeiro intermediário se tornando propícia a infecção por S. mansoni. Além disso, essa região caracteriza-se por apresentar faixas endêmicas para determinados tipos de parasitoses ${ }^{3,17,25}$. Dessa forma, o presente estudo tem como objetivo traçar o perfil epidemiológico da EM na região Sudeste do Brasil entre os anos de 2013 a 2017.

\section{Metodologia}

O Brasil é um país tropical e é formado por um conjunto de cinco regiões: Norte, Nordeste, Centro-Oeste, Sudeste e Sul. A região Sudeste, foco deste trabalho, é constituída pelos estados do Espírito Santo (ES), Minas Gerais (MG), Rio de Janeiro (RJ) e São Paulo (SP) e é a região mais habitada e a segunda menor em extensão territorial, compondo $12 \%$ do território brasileiro. A área territorial é de aproximadamente 925 mil km² e, em 2017, a população estimada foi de 86.949.714 habitantes, sendo considerada a região mais populosa do país ${ }^{25}$.

Para este estudo do tipo descritivo de tendência temporal, obtiveram-se os dados dos sistemas de informação em saúde, do qual utilizamos o Sistema de Informação de Agravos de Notificação (SINAN), considerando o período de 2013 a 2017, pois optou-se pelo recorte dos últimos cinco anos que estavam disponíveis durante o período da coleta em junho de 2020. Todas as informações foram recolhidas das notificações disponíveis no Departamento de Informática do Sistema Único de Saúde (DATASUS). O cálculo da incidência foi realizado a partir da estimativa da população projetada segundo o Instituto Brasileiro de Geografia e Estatística (IBGE).

A amostra do estudo é composta por todos os casos notificados de esquistossomose em residentes no estado de São Paulo, Minas Gerais, Rio de Janeiro e Espírito Santo, contabilizados a partir dos dados secundários oriundos do DATASUS, no período de 2013 a 2017, que contabilizou um total de 21.464 registros.

Os critérios de inclusão se basearam no número absoluto de casos notificados elegíveis de EM na região sudeste, tendo em vista que a análise dos dados obtidos por meio do DATASUS teve o objetivo de comparar com fontes bibliográficas a permanência dessa DNT em quatro estados brasileiros.

Um possível viés é o de informação devido a existência de subnotificações dos casos na base de dados, o que pode impactar na realidade da EM na região do estudo. Diante dos dados disponíveis no período analisado foram selecionadas as variáveis: casos confirmados, separados pelas cinco regiões do país e pelos estados da região Sudeste; população residente estimada, segundo o IBGE; faixa etária, separando os indivíduos em menores de um ano e em grupos com intervalo de dezenove anos; sexo; e zona de residência, sendo delimitada por área urbana, rural, periurbano e os casos em branco, sendo em seguida organizados em planilhas realizadas no Excel 2016, construindo-se tabelas e gráficos para a análise dos resultados.

Sobre os aspectos éticos, os dados obtidos por esse trabalho utilizaram fontes secundárias de saúde de base não nominal e disponíveis em um banco de domínio público do Ministério da Saúde, respeitando os princípios éticos da Resolução 466/2012 do Conselho Nacional de Saúde. Os dados foram verificados de forma integral, não ocasionando danos à população estudada e não sendo necessário a submissão desse estudo ao Comitê de Ética e Pesquisa.

\section{Resultados}

Entre os anos de 2013 a 2017, verificou-se um total de 28.781 casos confirmados de EM no Brasil. É possível observar uma redução dos casos totais no país, tendo a região Sudeste e Nordeste registrado os maiores índices de ocorrências notificadas $21.464 \quad(74,6 \%)$ e $6.399 \quad(22,2 \%)$, respectivamente (Tabela 1 ). 
De acordo com os dados obtidos do SINAN, a região Sudeste vem reduzindo o número de casos confirmados ao longo do tempo, diferentemente da região Nordeste que, em 2017, apontou um aumento nos casos notificados 1.220 (28\%) ao comparar com o ano de 2016 em que obteve 1.092 (21.2\%). Sobre os dados referentes as regiões Centro-Oeste e Sul, constata-se a pouca variação dos casos detectados de EM, porém na região Norte ocorreu um acréscimo de aproximadamente 40 casos em 2017 em relação ao ano de 2016 (Tabela 1).

Tabela 1 - Casos confirmados de esquistossomose mansônica no Brasil entre os anos de 2013 a 2017.

\begin{tabular}{|c|c|c|c|c|c|}
\hline AnolRegião & $\begin{array}{c}\text { Centro- } \\
\text { Oeste }\end{array}$ & Nordeste & Norte & Sudeste & Sul \\
\hline 2013 & 69 & 1185 & 42 & 5098 & 40 \\
\hline 2014 & 63 & 1419 & 70 & 4863 & 51 \\
\hline 2015 & 64 & 1483 & 59 & 4733 & 43 \\
\hline 2016 & 69 & 1092 & 64 & 3859 & 56 \\
\hline 2017 & 66 & 1220 & 100 & 2911 & 62 \\
\hline
\end{tabular}

Fonte: Ministério da saúde/SVS - Sistema de Informação de Agravos de Notificação - Sinam Net.

Percebe-se que nos estados da região Sudeste, entre o espaço temporal analisado, 2013 a 2017, os maiores valores absolutos de contaminação estão localizados em MG e SP com $15.724(73,25 \%)$ e $3.333(15,52 \%)$, respectivamente. Já o estado do ES apresentou $1.921(8,95 \%)$ casos dessa DNT, seguido pelo RJ com 486 (2,26\%). No ano de 2013, MG apontou
$3.646(71,51 \%)$ casos de infecção para EM, tendo ao longo do período diminuído o índice de infectados, registrando 1.941 (66,7\%) em 2017, contudo ainda representa a maior porcentagem dos casos de toda região (Figura 1).

Figura 1 - Casos Notificados de esquistossomose mansônica nos estados da região sudeste entre o período de 2013 a 2017

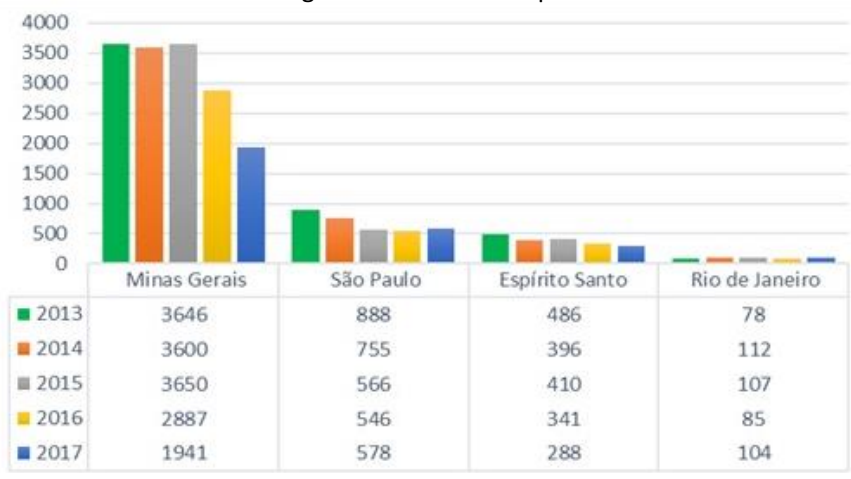

Fonte: Ministério da Saúde/SVS - Sistema de informação de agravos de Notificação - Sinan Net

Há uma queda na evolução histórica da EM na região Sudeste, em que a incidência (casos novos confirmados (população em risco x 10n) foi de 3,35 casos detectados para cada 100.000 habitantes em 2017, tendo em vista que em 2013 foi de 6,03. Além disso, nesses cinco anos analisados, MG e ES apresentaram a maior taxa de incidência, sendo que em 2017 o cálculo foi de 9,19 e 7,17, nessa ordem. Por outro lado, o RJ conta com um coeficiente de 0,62, possuindo o menor número de novos indivíduos contaminados pelo parasito (Figura 2).

Figura 2 - Taxa de incidência de esquistossomose mansônica (casos por 100.000 habitantes) na região sudeste de 2013 á 2017.

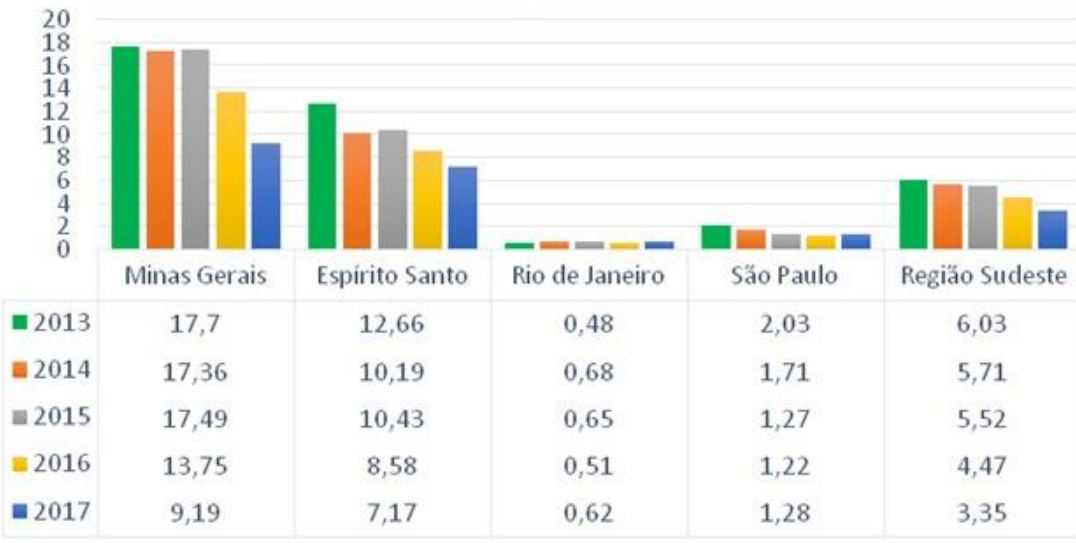

Fonte: Ministério da Saúde/SVS - Sistema de informação de Agravos de Notificação - Sinam Net 
Uma análise em relação a faixa etária mostrou que há relatos de infecção pelo $S$. mansoni, sendo a de 20 aos 39 anos com a frequência mais alta e responsável pelo registro de $1.034(35,5 \%)$ casos confirmados. Em relação ao sexo, o masculino foi o maior número de casos notificados com 1.840 (63,2\%) sobre $1.071(36,8 \%)$ do sexo feminino (Tabela 2).
A partir dos achados acerca da zona de residência, é nítida a predominância dessa parasitose nas áreas urbanas da região Sudeste, correspondendo a 2.117 (72,7\%) indivíduos detectados em 2017. Entretanto, há um equilíbrio dos dados ao observar o estado ES, que conta com 139 (48,3\%) casos na área rural e 143 (49,6\%) notificações na zona urbana, contrapondo o que se observa na região urbana dos outros estados (Tabela 2).

Tabela 2 - Distribuição dos casos confirmados de esquistossomose mansônica na região sudeste em 2017 pela faixa etária, sexo e zona de residência.

\begin{tabular}{|c|c|c|c|c|}
\hline Faixa etária & $\begin{array}{l}\text { Frequência } \\
\text { Absoluta }\end{array}$ & $\begin{array}{l}\text { Frequência } \\
\text { Relativa }\end{array}$ & $\begin{array}{l}\text { Frequência } \\
\text { Relativa (\%) }\end{array}$ & $\begin{array}{c}\text { Frequência Relativa } \\
\text { Acumulada (\%) }\end{array}$ \\
\hline$<1$ Ano & 22 & 0,0075 & 0,75 & 0,75 \\
\hline $1-19$ & 432 & 0,1484 & 14,84 & 15,59 \\
\hline $20-39$ & 1034 & 0,3552 & 35,52 & 51,11 \\
\hline $40-59$ & 983 & 0,3377 & 33,77 & 84.88 \\
\hline $60-79$ & 411 & 0,1412 & 14,12 & 99 \\
\hline $80>$ & 29 & 0,01 & 1 & 100 \\
\hline Total & 2911 & 1 & 100 & \\
\hline Sexo & $\begin{array}{l}\text { Número de } \\
\text { casos }\end{array}$ & & & \\
\hline Masculino & 1840 & & & \\
\hline Feminino & 1071 & & & \\
\hline Total & 2911 & & & \\
\hline Zona de Residência/ Estado & Minas Gerais & $\begin{array}{l}\text { Espirito } \\
\text { Santo }\end{array}$ & $\begin{array}{l}\text { Rio de } \\
\text { Janeiro }\end{array}$ & São Paulo \\
\hline Em branco & 40 & 5 & 4 & 24 \\
\hline Urbano & 1371 & 143 & 72 & 531 \\
\hline Rural & 508 & 139 & 26 & 20 \\
\hline Periurbano & 22 & 1 & 2 & 3 \\
\hline Total & 1941 & 288 & 104 & 578 \\
\hline
\end{tabular}

Fonte: Ministério da saúde/SVS - Sistema de informação de agravos de Notificação - Sinan Net 


\section{Discussão}

Tendo em vista que os casos de EM surgiram na região Nordeste do Brasil, nota-se que nas últimas décadas houve uma alteração nessas dinâmicas para outras localidades, devido à presença de fatores bióticos e abióticos, a busca pela oportunidade de emprego e o crescimento do ecoturismo. Essa colocação é corroborada por diferentes estudos e pela análise dos resultados deste trabalho ao indicar o surgimento de novos casos em outras áreas ${ }^{12,25,27,28}$.

De acordo com os resultados encontrados e com as pesquisas realizadas, constata-se que o ES, MG e SP apresentaram uma diminuição dos quadros dessa parasitose ${ }^{29,30,31,32}$. Possivelmente se justifica pelas ações estaduais, tendo como exemplo as intervenções adotadas pelo governo paulista, que dentre as estratégias de combate foi criada em 2009 a Semana da Esquistossomose que consiste em um programa educativo voltado para a orientação dos estudantes e da população sob o risco de contaminação ${ }^{33}$.Todavia, há o inverso ao analisar os dados do RJ, em que houve um crescimento no número de infectados, provavelmente ocasionado pela presença de fatores condicionantes e das intempéries locais, como observado no estudo aplicado ao bairro Alto da Boa Vista - RJ, que mostra os motivos determinantes para o incremento de novos casos locais, dentre eles: imigrantes de áreas endêmicas, o despejo de esgoto no rio local e a sua utilização para atividades de lazer ${ }^{34}$.

Cada estado da região Sudeste apresenta suas peculiaridades quando se trata das características físicas, ambientais e sociais, o que permite uma heterogeneidade de casos confirmados entre as diferentes localidades ${ }^{17,35}$. Considera-se como áreas endêmicas, ou seja, locais de risco para determinada doença o estado do ES e MG, tendo uma predominância no norte e no nordeste do estado mineiro ${ }^{36}$. A vertente de diferentes trabalhos e a realização deste levantamento epidemiológico apontam uma maior incidência nesses estados ao se comparar com RJ e SP, sendo justificada pela questão socioeconômica e pela presença dos focos de transmissão do S. mansoni nesses territórios ${ }^{37}$. Em contrapartida, o Inquérito Nacional publicado em 2018 por Naftale Katz, realizado em escolares de 7 a 17 anos, comprova uma maior proporção de casos nos estados de MG e RJ ${ }^{29}$.

A falta de educação sanitária, hábitos comportamentais e a ausência de saneamento básico contribuem para a ocorrência de casos infantis e em idosos. Entretanto, identifica-se que, entre os anos de 2013 a 2017, a faixa etária mais acometida é entre 20 a 39 anos e isso é decorrente da relação com o local de trabalho, o ecoturismo e ao lazer. Além disso, o maior número de infectados pelo $S$. mansoni é do sexo masculino, indo de encontro com as afirmativas de outros autores que abordam sobre o predomínio da transmissão entre homens adultos, associado na maioria das vezes a atividade econômica, como a agricultura, a pecuária e a pesca ${ }^{12,34,38}$.

Da mesma maneira que neste estudo, diferentes autores destacaram a predominância de EM na zona urbana. Isso é decorrente de como a dinâmica da doença é compreendida por meio do movimento migratório rural e da geografia do país, tendo a industrialização contribuído para a mudança do fluxo populacional rural-urbano, provocando um aumento demográfico desordenado no espaço e promovendo o surgimento de habitações precárias $^{12,39,40}$. Nesses locais, geralmente, não há saneamento básico, e a deposição do esgoto é a céu aberto ou em rios e lagos. Diferentemente do habitual, como se vê no estado do ES, há uma alteração na dinâmica das contaminações, em que a zona rural e a urbana se equiparam em casos confirmados. É notória a necessidade de cuidados com o surgimento de novas moradias, sobretudo as irregulares ${ }^{41}$.

A EM é uma enfermidade que danifica múltiplos órgãos, como o hepático e o esplênico, e causa mudanças sistêmicas no organismo humano, podendo resultar em óbito ${ }^{3}$. Diante de todo tratamento existente e das facilidades das técnicas diagnósticas, este estudo e outras publicações registram para a mesma questão, que as regiões Nordeste e Sudeste são consideradas expressivas em novos casos confirmados, tornando-se territórios endêmicos ${ }^{36,42}$.

\section{Considerações finais}

Diante do exposto, conclui-se que tanto os casos confirmados de EM quanto a incidência reduziram entre os anos de 2013 a 2017 no Sudeste do Brasil. A análise do perfil epidemiológico nessa região revela que predominaram as notificações do sexo masculino, principalmente na faixa etária entre 20 a 39 anos e que residem na zona urbana.

Uma limitação do presente trabalho é a presença de subnotificações dos casos no SINAN, 
na qual comprometem a real situação da EM na região estudada.

Portanto, destaca-se a importância dos dados coletados por este estudo, permitindo com que as figuras governamentais trabalhem com medidas profiláticas que combatam a doença, como o controle da proliferação do caramujo através de moluscicidas e que foquem em melhorias no saneamento básico, bem como na educação em saúde.

\section{Referências}

1. Prata A. Esquistossomose Mansoni. In: Veronesi R, Veronesi FR, (editor). Tratado de infectologia. 3. ed. São Paulo: Editora Atheneu; 2007. p.2015-2038.

2. Ministério da Saúde (BR). Secretaria de Vigilância em Saúde. Departamento de Vigilância Epidemiológica. Vigilância da Esquistossomose Mansoni: diretrizes técnicas. 4. ed. Brasília (DF): Ministério da Saúde; 2014..

3. Neves DP, Melo AL, Linardi PM, Vitor RWA. Parasitologia Humana. 13. ed. Rio de Janeiro: Atheneu; 2016. p.225-256.

4. Souza FPC, Vitorino RR, Costa AP, Faria FC Junior, Santana LA, Gomes AP. Esquistossomose mansônica: aspectos gerais, imunologia, patogênese e história natural. Ver Bras Clin Med 2011; São Paulo, 2011 julago;9(4):300-7.

5. World Health Organization. Neglected Tropical Diseases progress dashboard 2011-2020 [Internet]. [acesso em 18 Jun 2020]. Disponível em: https://www.who.int/neglected-tropicaldiseases-progress-dashboard-2011-2020.

6. World Health Organization. Schistosomiasis [Internet]. [acesso em 18 Jun 2020]. Disponível em: http://www.who.int/mediacentre/factsheets/fs1 15/en/.

7. World Health Organization. What is shistosomiasis? [Internet]. [acesso em 12 Jun 2020]. Disponível em: https://www.who.int/schistosomiasis/disease/en l.

8. WHO/PAHO - World Health Organization/Pan American Health Organization. Neglected infectious diseases in the Americas: success stories and innovation to reach the neediest. 2016. [Internet]. [acesso em 15 Jun 2020] Disponível em: https://iris.paho.org/handle/10665.2/31250.

9. Ministério da Saúde (BR). Secretaria de Vigilância em Saúde. Coordenação-Geral de Desenvolvimento da Epidemiologia em Serviços.
Guia de Vigilância em Saúde: volume único [recurso eletrônico] / Ministério da Saúde, Secretaria de Vigilância em Saúde, CoordenaçãoGeral de Desenvolvimento da Epidemiologia em Serviços. 3. ed. - Brasília: Ministério da Saúde, 2019.

10. Ministério da Saúde (BR). Secretaria de Vigilância em Saúde. Departamento de Vigilância das Doenças Transmissíveis. Educação em saúde para o controle da esquistossomose / Ministério da Saúde, Secretaria de Vigilância em Saúde, Departamento de Vigilância das Doenças Transmissíveis. - Brasília: Ministério da Saúde, 2018..

11. Reis M. Esquistossomose, pobreza e saneamento. [Dissertação]. São Paulo: Faculdade de Saúde Pública, Universidade de São Paulo; 2018.

12. Calasans TAS, Souza GTR, Melo CM, Madi RR, Jeraldo VDLS. Socioenvironmental factors associated with Schistosoma mansoni infection and intermediate hosts in an urban area of northeastern Brazil. PLoS ONE. 2018;13: e0195519.

13. Tibiriça SHC, Guimarães FB, Teixeira MTBA. A esquistossomose mansoni no contexto da política de saúde brasileira. Cienc. Saude Coletiva. 2011;16(1):1375-81.

14. Massara CL, Amaral GL, Caldeira RL, Drummond SC, Enk MJ, Carvalho OS. Esquistossomose em área de ecoturismo do Estado de Minas Gerais, Brasil. Cadernos de Saúde Pública. 2008; 24(7):1709-1712.

15. Oliveira DS, Santos VB, Melo AGS, Lima AS, Carvalho CD, Allegretti SM, et al. Schistosomiasis mansoni in urban Northeast Brazil: influence of rainfall regime on the population dynamics of Biomphalaria sp. Revista da Sociedade Brasileira de Medicina Tropical, 46(5), 654-657.

16. Vidal CHF, Gurgel FV, Ferreira MLB, Azevendo HRC Filho. Epidemiological aspects in neuroschistosomiasis. Arq Neuropsiquiatr 2010;68(1):72-5.

17. REY L. Parasitologia. 4. ed. Rio de Janeiro: Guanabara Koogan; 2008. p.435-499.

18. Vitorino RR, Souza FPC, Costa AP, Faria Júnior FC, Santana LA, Gomes AP. Esquistossomose mansônica: diagnóstico, tratamento, epidemiologia, profilaxia e controle. Rev Bras Clin Med 2012; 10(1):39-45. 
19. Chaves A, Alcantara OS, Carvalho OS, Santos JS. Estudo comparativo dos métodos coprológicos de Lutz, Kato-Katz e Faust modificado. Rev. Saude Publica 13: 348-352.

20. Barbosa CS, Gomes EC, Marcelino JM, Cavalcante KR, Nascimento WC. Quality control of the slides by Kato-Katz method for the parasitological diagnosis of schistosomiasis infection by Schistosoma mansoni. Jornal Brasileiro de Patologia e Medicina Laboratorial, 53, 110-114.

21. WHO/PAHO. Schistosomiasis Regional Meeting. Defining a road map toward verification of elimination of schistosomiasis transmission in Latin America and the Caribbean by 2020. [Internet]. [Acesso em 15 de Agos 2020]. Disponível

em:

http://www.PAHO.org/hq/index.php?option=co

m_docman\&task=doc_download\&ltemid=270\&gi $\mathrm{d}=28841$ \&lang=en.

22. Kongs $A$, Marks $G$, Verlé $P$, Van der Stuyft P. The unreliability of the Kato-Katz technique limits its usefulness for evaluating $S$. mansoni infections. Trop. Med Int Health. 2001 Mar;6(3):163-9. doi: 10.1046/j.13653156.2001.00687.

23. Oliveira WJ, Magalhães FDC, Elias AMS, Castro VN, Favero V, Oliveira $A A$, et al. (2018) Evaluation of diagnostic methods for the detection of intestinal schistosomiasis in endemic areas with low parasite loads: Saline gradient, Helmintex, Kato-Katz and rapid urine test. PLOS Neglected Tropical Diseases 12(2): e0006232. doi.org/10.1371/journal.pntd.000623 24. Ministério da Saúde: Relação Nacional de Medicamentos Essenciais - Rename [Internet]. [acesso em 13 Agos 2020]. Disponível em: https://saude.gov.br/saude-de-a-z/rename.

25. Instituto Brasileiro de Geografia e Estatística [Internet]. [acesso em 14 Jul 2020]. Disponível em: https://www.ibge.gov.br/.

26. Enk MJ, Lima AL, Barros HS, Massara CL, Coelho PZ, Schall VT. Fatores relacionados à transmissão e infecção por Schistosoma mansoni em um vilarejo da região sudeste do Brasil. Mem. Inst. Oswaldo Cruz [Internet]. [acesso em 13 de Jun 2020]. Disponível em: http://www.scielo.br/scielo.php?script=sci_artte xt\&pid=S007402762010000400037\&lng=en. doi: 10.1590/S0074-02762010000400037.

27. Pellegrini AF, Remos C, Ribeiro J. A medicina comunitária, a questão urbana e a marginalidade. Rev. Saúde e medicina no Brasil. 1978.

28. Blanton RE, Barbosa LM, Reis EA, Carmo TM, Santos CR, Costa JM. A contribuição relativa da imigração ou aumento local para a persistência da esquistossomose urbana em Salvador, Bahia, Brasil. PLoS Negl Trop Dis. 2015; 9 (3): e0003521.

29. Katz N, compiler. Inquérito Nacional de Prevalência da Esquistossomose mansoni e Geohelmintoses [bibliografia]. 22. ed. Belo Horizonte: Centro de Pesquisa René Rachou; 2018.

30- Spala MR. Esquistossomose no Espírito Santo [Monografia]. Vitoria: Universidade Federal do Espírito Santo; 2013.

30. Spala MR. Esquistossomose no Espírito Santo [Monografia]. Vitoria: Universidade Federal do Espírito Santo; 2013.

31. Drummond SC, Pereira SR, Silva LC, Antunes CM, Lambertucci JR. Schistosomiasis control program in the state of Minas Gerais in Brazil. Mem. Inst. Oswaldo Cruz [Internet]. [acesso em 20 Agos 2020]. Disponível em: http://www.scielo.br/scielo.php?script=sci_artte xt\&pid=S007402762010000400029\&Ing=en. doi: 10.1590/S0074-02762010000400029.

32. Souza D, Ciaravolo $R$, Kanamura $H$, Gargioni C, MacDowell A, Falcão C, et al. Esquistossomose Mansônica no Estado de São Paulo: Aspectos Epidemiológicos.

33. Estado de São Paulo implanta Semana da Esquistossomose. BEPA, Bol. epidemiol. paul. [Internet]. [acesso 22 Setem 2020]. Disponível em:

http://periodicos.ses.sp.bvs.br/scielo.php?script= sci_arttext\&pid=S180642722009000400004\&lng $=$ pt.

34. FC Nunes, Costa ME, Filhote MI, Sharapinn M. Perfil epidemiológico da esquistossomose mansoni no bairro alto da Boa Vista, Rio de Janeiro. Cadernos de Saúde Coletiva. 2005.

35. Hotez PJ, Fujiwara RT. Brazil's neglected tropical diseases: an overview and a report card. Microbes Infect. 2014 Ago;16(8):601-6. doi: 10.1016/j.micinf.2014.07.006.

36. Ministério da Saúde: Esquistossomose: causas, sintomas, tratamento, diagnóstico e prevenção [Internet]. [acesso em 1 de Jul de 2020]. Disponível em: http://www.saude.gov.br/saude-de-az/esquistossomose.

37. Barbosa, CS, Favre TC, Amaral RS, Pieri OS. Epidemiologia e controle da Esquistossomose mansoni. In: CARVALHO, OS., COELHO, PMZ., and LENZI, HL., orgs. Schitosoma mansoni e esquistossomose: uma visão multidisciplinar. Rio de Janeiro: Editora FIOCRUZ, 2008. p 964-1008.

38. Rocha JM, Santos MC, Lima MV, Calheiros CM, Wanderley FS. Aspectos epidemiológicos e distribuição dos casos de 
infecção pelo Schistosoma mansoni em municípios do Estado de Alagoas, Brasil. Rev PanAmaz Saude [Internet]. [acesso em 10 Agos 2020]. Disponível em: http://scielo.iec.gov.br/scielo.php?script=sci_artt ext\&pid=S217662232016000200027\&lng=pt. htt p://dx.doi.org/10.5123/S217662232016000200003.

39. Barbosa CS, Souza AT, Leal-Neto OB, Gomes CS, Araujo KC, Gomes M, et al. Turismo de risco para esquistossomose mansônica em Porto de Galinhas, Estado de Pernambuco, Brasil. Ver. Pan-Amaz. Saude [Internet]. [acesso em 13 Agos 2020]. Disponívelem: http://scielo.iec.gov.br/scielo.php?script=sci_artt ext\&pid=S217662232015000300007\&lng=pt.

40. Amorim MN, Rabello A, Contreras RL, Katz N. Epidemiological characteristics of Schistosoma mansoni infection in rural and urban endemic areas of Minas Gerais, Brazil. Mem Inst Oswaldo Cruz. 1997 Sep-Oct;92(5):577-80. doi: 10.1590/s0074-02761997000500001.

41. Melo AGS, Melo CM, Oliveira CCC, Oliveira DS, Santos VB, Jeraldo VLS. Esquistossomose em área de transição ruralurbana: reflexões epidemiológicas. Cienc. Cuid. Saúde [Internet]. [acesso 13 de Agos 2020]. Disponível em: http://periodicos.uem.br/ojs/index.php/CiencCui dSaude/article/view/12479. doi: 10.4025/cienccuidsaude.v10i3.12479.

42. Martins MFR, Pinheiro MC, Ramos ANJr, Alencar $\mathrm{CH}$, Bezerra FS, Heukelbach J. Trends in schistosomiasis-related mortality in Brazil, 20002011. Int J Parasitol. 2014 Dec;44(14):1055-62. doi: 10.1016/j.ijpara.2014.07.009.

\section{Endereço para Correspondência}

João Victor Barreto Costa

Av. 7 de setembro, 1793, apto 302, Esplanada.

Governador Valadares/MG.

CEP: 35010-171

E-mail: jv barreto@id.uff.br

Recebido em 23/04/2021

Aprovado em 10/08/2021

Publicado em 27/09/2021 